\section{(1) \\ FEB 241989}

\title{
Towards an Understanding of the Nuclear Potential ${ }^{1}$
}

\author{
David G. Richards \\ Physics Department \\ University of Edinburgh \\ The King's Buildings \\ Edinburgh EH9 3JZ, Scotland
}

ANL-HEP-CP $-88-93$

DE89 007260

\author{
Don K. Sinclair, Dennis Sive:- \\ High Energy Physics Division \\ Argonne National Iaboratory \\ Argonne, IL 60439, USA
}

\begin{abstract}
The formalism for investigating the $\bar{Q} q \bar{Q} q$ system on the lattice is constructed. We describe how the model may be used to study the nuclear potential, and present some preliminary results on the range of the nuclear force.
\end{abstract}

\section{DISCLAIMER}

\begin{abstract}
This report was prepared as an account of work sponsored by an agency of the United States Government. Neither the United States Government nor any agency thereof, nor any of their employees, makes any warsanty, express or implied, or assumes any legal liability or responsibility for the accuracy, completeness, or usefulness of any information, apparatus, product, or process disclosed, or represents that its use would not infringe privately owned rights. Reference herein to any specific commercial product, process, or service by trade name, trademark. manufacturer, or otherwise does not necessarily constitute or imply its endorsement, recommendation, or favoring by the United States Government or any agency thereof. The views and opinions of authors expressed herein do not necessarily state or reflect those of the United States Government or any agency thereof.
\end{abstract}

\footnotetext{
${ }^{1}$ work supported by the U.S. Department of Energy, Div 31-109-ENG-38.
} 
David G. RICHARDS; Don K. SINCLAIR, Dennis SIVERS

High Energy Physics Division, Argonne National Laboratory, Argonne, Illinois, 60439

The formalism for investigating the $\bar{Q}_{q} \bar{Q}_{q}$ system on the lattice is constructed. We describe how the model may be used to study the nuclear potential, and present some preliminary results on the range of the nucleat force

\section{INTRODUCTION}

Lattice gauge theory has already told us much about the low energy properties of QCD. We have reasonable estimates of hadronic masses and, as we have heard at this conference, there are now many calculations of phenomenological quantities such as the weak matrix elements and hadronic structure functions. However the latter measurements are really applicable to high energy processes, where factorization allows us to separate the calculation of the "hard", high mornentum transfer subprocess from the "soft" low momentum transfer matrix element. We have as yet little information concerning low energy hadronic interactions and in nuclear properties.

Nuclear physics is presumably described by the QCD Lagrangian. However nuclear interactions exhibit a couple of features that would seem difficult to ascribe to QCD. Firstly, why do nucleons in heavy nuclei retain their identity, even though the separations between the partons within a particular nucleon may be no greater than the separation between partons in two different nucleons. Secondly, how can QCD account for "colour saturation", whereby the binding energy between nucleons, or between colour singlet states in general, is very much less than the typical QCD energy scale. The aim of the exercise that I shall be describing today is to see whether a lattice simulation can shed some light on these sorts of issues. This work is very much of an expioratory nature, and a more complete study will appear

work supported by the U.S. Department of Energy, later. ${ }^{1}$

The simplest multiquark system that can exhibit non-trivial colour saturation is the $\bar{Q}_{a} Q_{b} \bar{Q}_{c} Q_{d}$ system, where the subscripts denote (not necessarily distinct) quark flavours. The four partons can form colour singlet mesons in two ways, $\left(\bar{Q}_{a} Q_{b}\right)\left(\bar{Q}_{c} Q_{d}\right)$ and $\left(\bar{Q}_{c} Q_{b}\right)\left(\bar{Q}_{a} Q_{d}\right)$. For the case where the $Q^{\prime} s$ are heavy quarks, the system has been investigated in the strong coupling approximation by Matsuoka and Sivers. ${ }^{2}$ They found that the mixing between the two different coloursinglet configurations is small, and that there is a lower energy state in which the $\bar{Q} Q$ pairs are indeed confined into hadrons.

The model of a nuclear "molecule" described above is clearly flawed, since the forces between colour singlet states can be due only to colour-flux tubes. The internucleon force is usually envisioned as being mediated through meson exchange. To accord with this picture we must allow for the exchange of light quarks g. An investigation of the full six-quark $N N$ system would be a monumental task. Instead we shall appeal to the quark-diquark picture of the nucleon, and explore the $\bar{Q}_{a} q_{b} \bar{Q}_{c} q_{d}$ system, where the subscripts denote the quark flavours, and in which the heavy antiquarks assume the rôle of the diquarks.

The rest of this talk is organised as follows. In Section III shall discuss the formulation of $\bar{Q} q \bar{Q} q$ systems on the lattice, and discuss some simple expectations for the model. In Section III I shall present our numerical results, and lay out a strategy for future investigations. ENG-38.

†Talk Presented at the International Symposium Lattice '88, Batavia, IL, USA, September 22-25, 1988.

'Address after the 1st. October: Dept. of Physics, University of Edinburgh, The King's Buildings, Edinburgh, Scotland. 


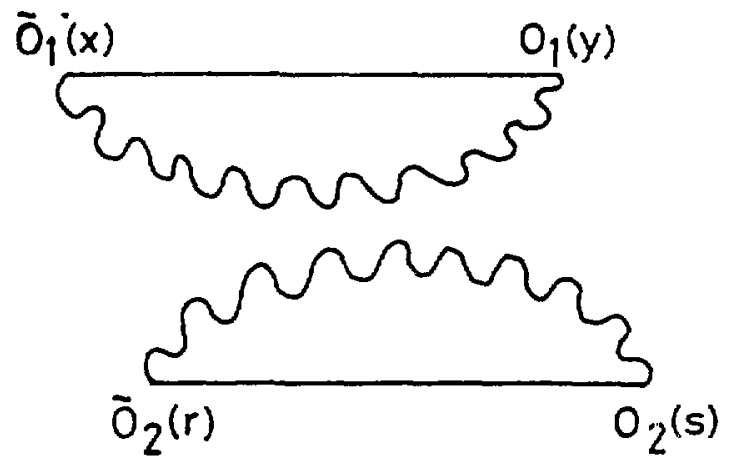

Figure 1: Diagram contributing to flavour non-exchange part of nuclear potential

\section{2. $\bar{Q}_{q} \bar{Q}_{q}$ SYSTEMS ON THE LATTICE}

$\bar{Q} q$ systems have been studied extensively by Eichten et al. ${ }^{3}$ in the regime $m_{q} \leq \Lambda_{Q C D} \ll m_{Q}$. Since the momentum transfer between the heavy quark and the light quark is typically of the order of $\Lambda_{Q C D}$. and very much less than $m_{Q}$, the heavy quark can be treated non-relavistically, and its propagator expanded as a power series in $m_{Q}^{-1}$. The technique has been applied to measurements of heavy-light meson masses and decay constants, and should be a particularly appropriate way of treating the $T$ meson. We shall apply the same techniques to the study of the $\bar{Q} q \bar{Q} q$ system, but shall always work to lowest order in $m_{Q}^{-1}$.

The heavy quark propagator is given by

$$
\begin{aligned}
S_{H}(r ; s)= & P(r ; s)\left[\theta\left(s_{0}-r_{0}\right) e^{-m_{Q}\left(s_{0}-r_{0}\right)} \gamma_{-}\right. \\
& \left.+\theta\left(r_{0}-s_{0}\right) e^{-m_{Q}\left(r_{0}-s_{0}\right)} \gamma_{+}\right],
\end{aligned}
$$

where

$$
P(r ; s)=U_{4}\left(r, r_{0}\right) \ldots U_{4}\left(\mathrm{r}, s_{0}-1\right)
$$

for $r_{0}<s_{0}$, with a similar expression for $r_{0}>s_{0} . \gamma_{+}$ and $\gamma$ - denote the positive and negative projection operators respectively. It should be noted that on a periodic lattice there is an additional contribution to the propagator that arises from the Wilson line that passes in the opposite direction around the lattice. This contribution is exponentially suppressed in $m_{Q}$ providing the Wilson line is restricted to less than haif the time dimension of the lattice. The light quark propagators are computed in the usual way.

The diagrams contributing to the internucleon potential are shown in Fig. 1 and Fig. 2; the solid and curly

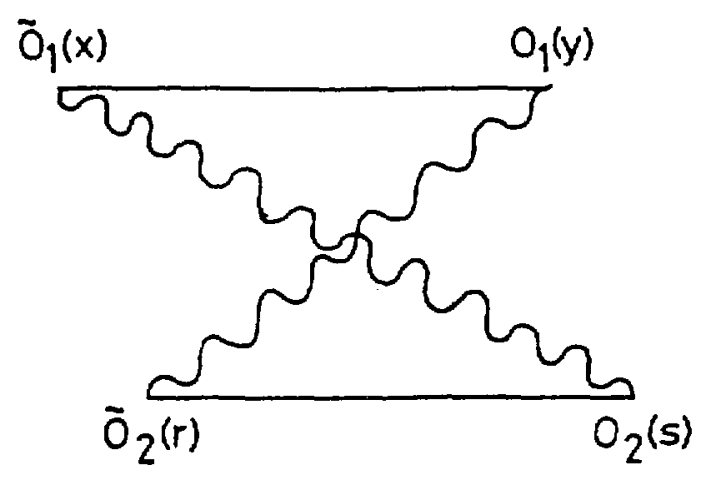

Figure 2: Diagram contributing to flavour exchange part of nuclear potential

lines denote the heavy and light quark propagators respectively. Their evaluation should allow us to extract the range of the nuclear force (in particular whether the range depends on the $t$-channel quantum numbers), and ultimately the strength of the nuclear potential.

We employ local interpolating operators for the heavy-light mesons

$$
O(x)=\bar{\psi}_{a}(x) \Gamma_{A} \Psi_{H}(x)
$$

where $\Gamma_{A}=\gamma_{B}$ (Pseudoscalar) or $\Gamma_{A}=\gamma_{i}$ (Vector). We begin with the construction of the correlator

$$
C(x, r ; y, s)=\left\{\tilde{O}_{1}^{\dagger}(x) \tilde{O}_{2}^{\dagger}(r) O_{1}(y) O_{2}(s)\right\},
$$

where $y_{0}>x_{0}$ and $s_{0}>r_{0}$. In order to extract the range of the force between the mesons we form the " $z$ sliced" correlator

$$
\bar{C}(R, T)=\sum_{t_{1}, t_{2}, \mathbf{x}_{\perp}} C(x, r ; y, s) .
$$

where

$$
\begin{aligned}
& x=\left(0, t_{1}\right), \\
& y=\left(0, t_{1}+T\right) . \\
& r=\left(\mathbf{a}_{\perp}, R, t_{2}\right), \\
& s=\left(\mathbf{a}_{1}, R, t_{2}+T\right) .
\end{aligned}
$$

The contribution of the flavour exchange diagram to $\bar{C}$ can be calculated by computing the quark propagator from one point on each time slice to every point on the lattice. However for the disconnected diagram the propagators from every point on one time slice to 
every point on the lattice have to be calculated. This is clearly a very much more demanding task, and therefore as a first step I shall restrict the discussion to the flavour exchange contribution. Even this more limited calculation should still allow us to measure the range of the internucleon force.

At large separations $R$ the correlator is dominated by the contribution of the lightest state $|n\rangle$ coupling to the operators in the $t$-channel and becomes,

$$
e^{-m R}\left\langle 0\left|\tilde{O}_{1}^{\dagger}(\mathbf{0}, 0) O_{1}(\mathbf{0}, T)\right| n\right\rangle\left\langle n\left|\tilde{O}_{2}^{\dagger}(\mathbf{0}, 0) O_{2}(\mathbf{0}, T)\right| 0\right\rangle .
$$

Before proceeding to the numerical results, it is worth discussing what our expectations are for the $t$-channel masses. I shall use the notation $V$ and $P$ to denote vector and pseudoscalar mesons respectively. The first process that we shall consider is

$$
\begin{aligned}
& P P \quad \longrightarrow P P \quad(s-\text { channel }) \\
& P P \quad \longrightarrow P P \quad(t-\text { channel }) .
\end{aligned}
$$

The particle exchanged in the $t$-channel has natural parity, and hence we expect the mass of the intermediate state in Eq. (2.7) to be that of the $\rho$. In order to see the exchange of a $\pi$ we must consider the process

$$
\begin{aligned}
& P V \rightarrow V P \\
& P V \longrightarrow V P \quad(s-\text { channel }) \\
&
\end{aligned}
$$

for which a particle of unnatural parity can be exchanged.

\section{NUMERICAL RESULTS AND CONCLUSIONS}

The gauge configurations were generated by Grady, Sinclair and Kogut ${ }^{4}$ on an $8^{3} \times 16$ lattice using the hybrid algorithm with four flavours of staggered fermion. However Wilson fermion propagators are employed in calculations of the spectrum 5 and the work $I$ am describing here, with the hoppling parameter chosen so that the pion mass obtained using hadron correlators constructed from Wilson fermion propagators is the same (in units of the lattice spacing) as that obtained when purely using staggered fermions. For this calculation the gauge configurations were replicated in the $z$ direction to yield an $8^{2} \times 16 \times 16$ lattice since, we are aiming to extract the lightest particle exchanged in that direction. Periodic boundary conditions were employed

\begin{tabular}{|c|c|c|c|c|}
\hline$O$ & $\pi_{1}$ & $\pi_{2}$ & $\rho_{1}$ & $\rho_{2}$ \\
\hline$M$ & $0.84(1)$ & $0.82(2)$ & $0.90(1)$ & $0.90(2)$ \\
\hline
\end{tabular}

Table 1: The mass $M$ obtained using operator $O$ on the unreplicated lattice

in the spatial directions, but an antiperiodic boundary condition was used in the time direction. The propagators were generated using a conjugate residual algorithm preconditioned according to the prescription of Yoshie et al. ${ }^{6} ;$ the large number of propagator evaluations ( 16 per configuration) made some form of preconditioning almost essential in this case. On the CRAY XMP/14 at Argonne National Laboratory the generation of the propagators required approximately 5 hours per configuration, with a further hour required to construct the correlators. The results presented today are obtained from an analysis of 19 configurations at $m=0.1$ and $\beta=5.4$, yielding a pion mass (on the unreplicated lattice) of $m_{x}=0.84$. As a reference point for the discussion the vector and pseudoscalar meson masses obtained on the unreplicated lattice are shown in $\mathrm{Ta}$ ble $1 .^{5}$

We have evaluated the correlators corresponding to the processes of equations (2.8) and (2.9) for a range of $R$ and $T$. The effective masses obtained at $T=1$, $T=3$ and $T=5$ are shown in Fig. $3(P P \longrightarrow P P)$ and Fig. $4(P V \longrightarrow V P)$. In each figure the masses of the $\pi$ and of the $\rho$ on the unreplicated lattice are shown as the dashed and dot-dashed line respectively. The estimates obtained for the masses of the particles exchanged in the $t$-channel are rather lower than the values for the $\pi$ and $\rho$ masses quoted in Table 1 . However it should be borne in mind that the latter results were obtained on the unreplicated lattice with different boundary conditions; that the values obtained in this calculation are rather lower is to be expected. It is not possible to distinguish with any degree of confidence between the masses of the natural parity $(\rho)$ and unnatural parity $(\pi)$ states. Finally it can be seen that the effective mass increases with increasing $R$ at larger values of $T$. This is presumable indicative of a repulsive force at short distances.

I have described a model that should prove a useful tool for studying the nuclear force within the frame- 

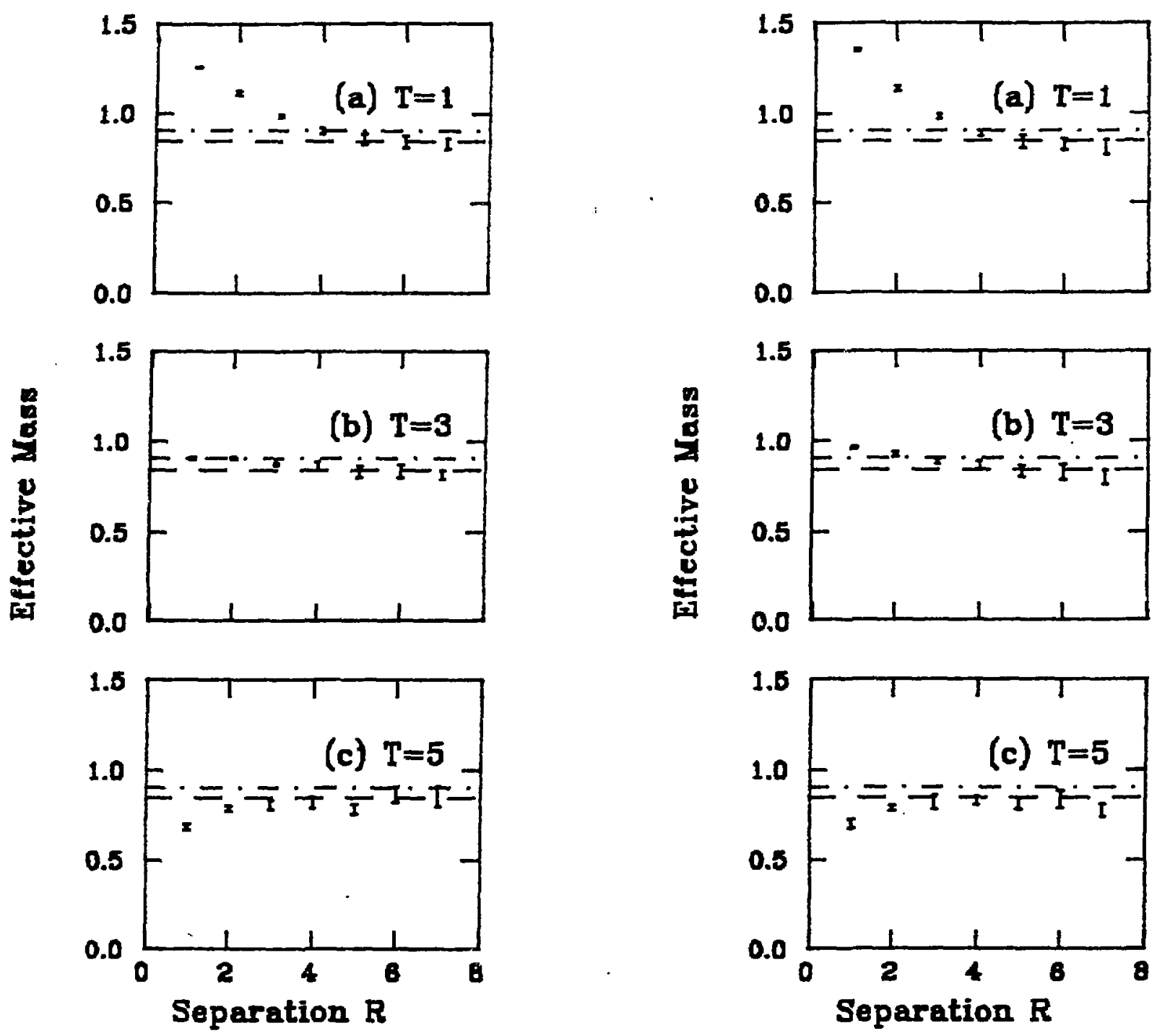

Figure 3: The effective mass of the particle mediating Figure 4: The effective mass of the particle mediating the nuclear force in the process $P P \longrightarrow P P$ is shown the nuclear force in the process $P V \longrightarrow V P$ is shown for separations $T=1, T=3$ and $T=5$ for separations $T=1, T=3$ and $T=5$ 
work of QCD. The results I have presented today are REFERENCES

very preliminary, and represent only the first step in a continuing study. We are presently obtaining a neasurement of the mass spectrum on the $8^{2} \times 16^{2}$ lattice, and repeating the calculation to lower values of the pion mass. It is only when these tasks have been performed that we expect to be able to verify whether the quantum numbers of the particles exchanged in the $t$-channel do indeed accord with our expectation. We shall then extend the project to address the question of measuring the strength of the internucleon potential.

\section{ACKNOWLEDGEMENTS}

We are very grateful to the National Center for Supercomputing Applications and to Argonne National Laboratory for use of their CRAYs. We are particularly indebted to the Computing Consulting staff at Argonne for the enthusiastic way in which they tackled many of the technical problems that arose.

1. D.Richards, D.Sinclair and D.Sivers, in preparation.

2. H.Matsuoka and D.Sivers, Phys. Rev. D33 (1986) 1441.

3. E. Eichten, talk at Intern. Symp. on Field Theory on the Lattice (Seillac,France, September-October 1987), Nuclear Physics B (Proc. Suppl. 4) (1988) 170.

4. M.P.Grady, D.K.Sinclair and J.Kogut, Phys. Lett. $200 B$ (1988) 149; D. K. Sinclair, talk at Intern. Symp. on Field Theory on the Lattice (Seillac, France, September-October 1987), Nuclear Physics B (Proc. Suppl. 4) (1988) 225.

5. D.G.Richards, Phys. Lett. 206B (1988) 107; D.G.Richards, talk at Intern. Symp. on Field Theory on the Lattice (Seillac, France, SeptemberOctober 1987), Nuclear Physics B (Proc. Suppl. 4) (1988) 221.

6. Y.Oyanagi, Comput. Phys. Comm. 42 (1986) 333. 